\title{
Isolated Nail Dystrophy: An Unlikely Presentation of Metastatic Renal Cell Carcinoma
}

\author{
Sarah F. McClees ${ }^{a} \quad$ James Robert Duncan $^{b} \quad$ Roger Ceilley $^{c}$ Tiffani L. Milless $^{d}$ \\ Boni E. Elewski ${ }^{\text {b }}$ \\ a School of Medicine, University of Alabama Birmingham, Birmingham, AL, USA; ${ }^{b}$ Department of Dermatology, \\ University of Alabama Birmingham, Birmingham, AL, USA; 'Dermatology, P.C., West Des Moines, IA, USA; \\ dlowa Pathology Associates, Des Moines, IA, USA
}

\section{Established Facts}

- Cutaneous metastases are rarely the initial presentation of an undiagnosed tumor in a previously malignancy-free individual. Metastasis of any malignancy to the nail unit is uncommon, and only a handful of cases of subungual renal cell carcinoma metastasis have been reported.

\section{Novel Insights}

- We describe a case of isolated nail dystrophy that proved to be the presenting symptom of a previously undetected renal cell carcinoma.

\section{Keywords}

Renal cell carcinoma $\cdot$ Nail bed metastasis $\cdot$ Nail tumor

\begin{abstract}
Metastasis of any malignancy to the nail unit is uncommon, and only a handful of cases of subungual renal cell carcinoma (RCC) metastasis have been reported. We describe a case of isolated nail dystrophy that proved to be the presenting symptom of a previously undetected RCC. In a patient presenting with a subungual lesion, tumor metastasis to the nail unit should be included in the clinical differential diagnosis in both oncology patients and previously cancer-free individuals, as a subungual metastasis may be the first indication of a clinically silent visceral malignancy.
\end{abstract}

(c) 2018 S. Karger AG, Basel

\section{KARGER}

(c) 2018 S. Karger AG, Basel

E-Mail karger@karger.com

www.karger.com/sad

\section{Introduction}

Metastases represent $2 \%$ of all cutaneous tumors and may be the first indication of advanced cancer or cancer recurrence [1]. Of all cutaneous metastases, renal cell carcinoma (RCC) is the primary tumor in $6-6.8 \%$ of cases $[2,3]$. Lesions typically present on the head or scalp, upper extremities, chest, neck, or nephrectomy scar [1]. Metastasis of any malignancy to the subungual area of the nail unit, which includes the nail matrix and nail bed, is uncommon. Previously, only a handful of cases of subungual RCC metastasis have been reported [4]. We describe a case of isolated nail dystrophy that proved to be the presenting symptom of a previously undetected RCC. 


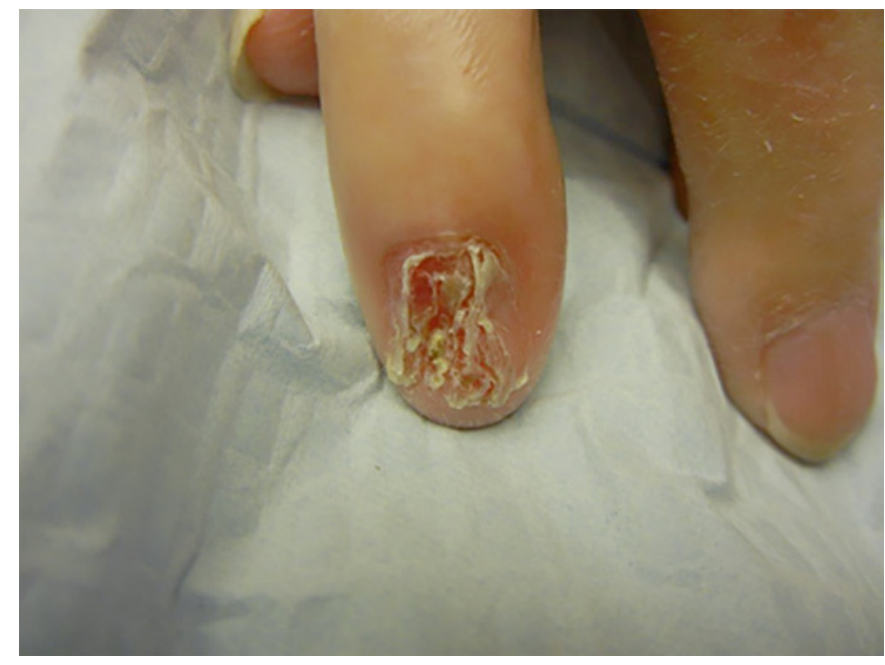

Fig. 1. Clinical characteristics. Isolated dystrophic appearance of the left index fingernail serving as the initial presentation of a previously unknown primary clear cell renal cell carcinoma.

\section{Case Report}

A 71-year-old female presented to the dermatology clinic as a referral from her primary care provider for a left index finger nail lesion. Four months prior to presentation, she experienced a direct trauma to the nail which led to persistent bleeding. This required treatment in the emergency department consisting of nail avulsion and cautery. Following this, she had dystrophic regrowth of the nail. On exam, she exhibited significant onychodystrophy isolated to her left index finger along with an apparent erythematous subungual mass (Fig. 1). The mass was initially thought to be a mxyoid cyst and was injected with intralesional triamcinolone $5 \mathrm{mg} / \mathrm{mL}$. The injection led to profuse bleeding. Due to the difficulty in achieving hemostasis, a saucerization biopsy of the proximal nail bed was performed, followed by debridement of the nail with curettage of the base. Examination of the hematoxylin and eosin-stained slides revealed a clear cell neoplasm with a prominent vasculature and areas of hemorrhage (Fig. 2). A panel of immunohistochemical stains was performed, with a differential diagnosis of metastatic RCC, melanoma, clear cell squamous cell carcinoma, and sebaceous carcinoma. Immunohistochemistry was positive for CD10, PAX-8, AE1/ $\mathrm{AE} 3$, and adipophilin, and negative for CK 5/6, p63, SOX-10, panmelanocytic cocktail, and GATA-3 (Fig. 2a-f). CK 5/6 and p63 are markers for squamous cell carcinoma, SOX-10 and pan-melanocytic cocktail are markers for melanoma, and adipophilin is positive in sebaceous lesions but can also be a sensitive marker for RCC $[5,6]$. CD10 is a metallomembrane endopeptidase that is highly sensitive but not specific for RCC (Fig. 2e), and PAX8 is a transcription factor that is sensitive and specific for RCC (Fig. 2f) [6]. Based on the immunohistochemistry with positive CD10, PAX8, AE1/AE3, and negative GATA-3, the diagnosis of metastatic RCC was made. Subsequent imaging revealed bilateral renal and adrenal masses with metastasis to the right lung. She was diagnosed with stage IV clear cell RCC and was referred to medical oncology, where she was started on palliative treatment with pazopanib.

\section{Discussion}

The overall incidence of metastasis to the skin from visceral malignancies is small, ranging from 0.6 to $10.4 \%$ of all patients with cancer [7]. Primary malignancies that most frequently metastasize to the skin are breast $(32.7 \%$ of all skin metastases seen), followed by bronchus and lung (13.2\%), skin (most commonly melanoma; 9.5\%), lymph nodes (7.5\%), oral cavity/pharynx/larynx (6.2\%), blood and bone marrow (5.5\%), and colorectal (4.2\%) [8] . Urologic cancers are less likely to metastasize to the skin compared to other primary malignancies, with a reported $1.1 \%$ of urologic malignancies metastasizing to skin [3]. Of the genitourinary malignancies, RCC is the most common to metastasize to skin, with the most common sites being the trunk and scalp [9].

Even more rare than cutaneous metastases are metastases to the nail unit. In a review of 133 patients with subungual metastases, the most common primary tumor site was the lung (41\%), followed by the kidney (11\%) and the breast (9\%) [4]. Subungual metastases generally present as a painful lesion of the distal digit. These lesions are often prone to bleeding, fluctuant, pulsatile, or perceived as hot. The most common appearance is either an enlargement or swelling of the distal digit, or a red to violaceous nodule that distorts the nail plate, the soft tissue of the distal digit, or both [4].

In a series of eight cases of RCC with subungual metastases, the median age at diagnoses of the primary carcinoma was 54 years (range 45-65). The subungual metastases occurred a median of 3 years later (range 4 months to 10 years). Signs and symptoms of the metastatic lesions included tenderness, nail bed dystrophy or discoloration, shortening of the nail plate, bleeding and ulceration, and malodorous discharge. Six of the eight cases involved a single digit. Bony metastasis in the involved digit was identified by radiographic evidence in five patients [10].

Cutaneous metastases are rarely the initial presentation of an undiagnosed tumor in a previously malignancy-free individual, occurring in less than $1.0 \%$ of oncology patients [11]. However, it is not uncommon for a metastatic subungual tumor to be the first sign of previously undiagnosed malignancy. In a review of 50 patients, subungual metastases were the first sign of a previously unknown primary malignancy in $44 \%$ of cases [4]. Diagnosis of subungual metastases can be challenging, especially if occurring in patients without a known primary malignancy. Clinical presentations can vary and frequently mimic other conditions, including infectious processes such as an abscess, paronychia, or osteo- 

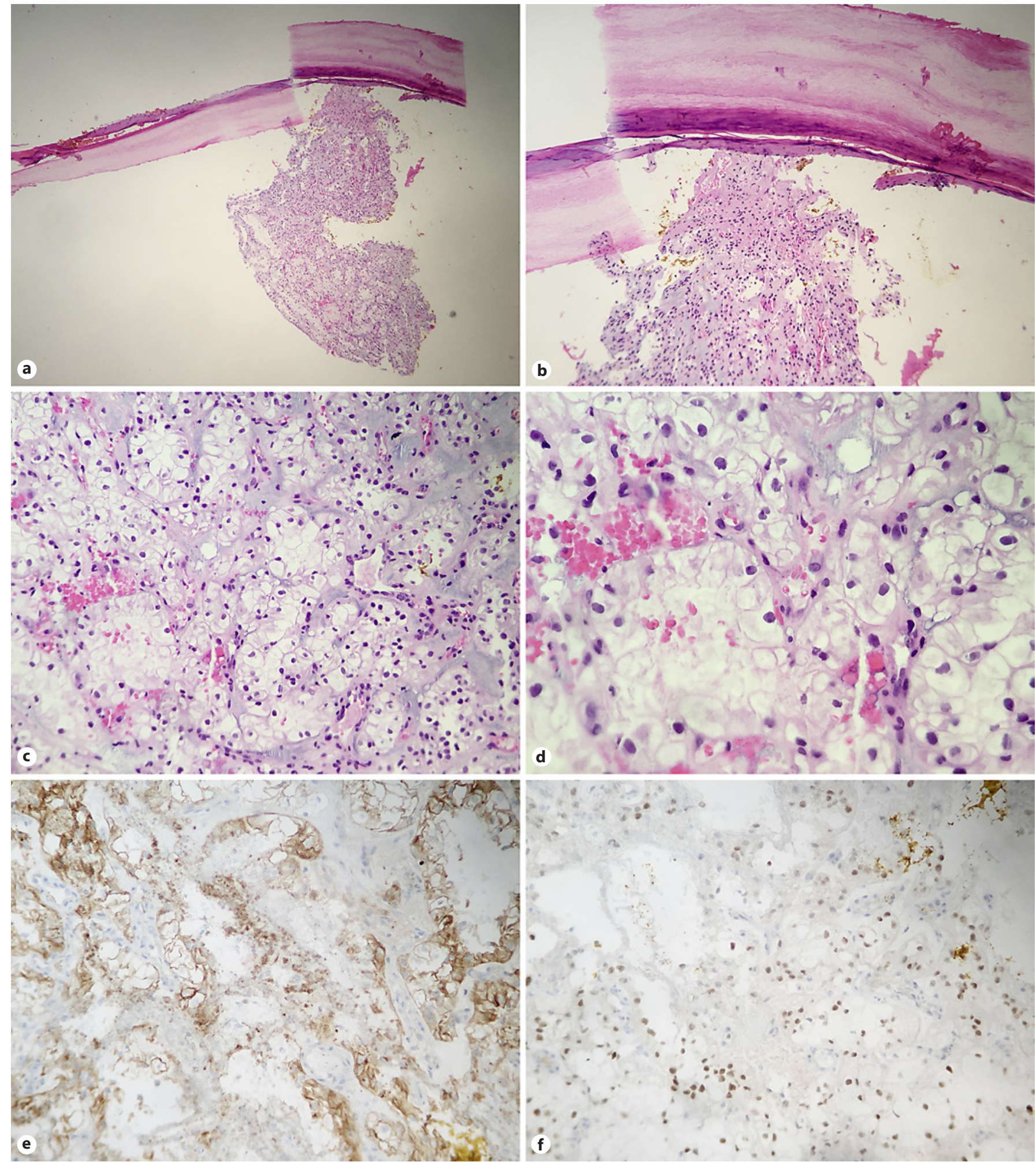

Fig. 2. Histopathology. Microscopic examination of a saucerization biopsy from the proximal nail bed of the left index fingernail demonstrates pathologic changes of metastatic renal cell carcinoma. Low- (a), medium- (b, c), and high-magnification (d) views reveal a malignant clear cell neoplasm associated with prominent

vasculature and areas of hemorrhage (hematoxylin and eosin; magnification: a $4 \times$, b, c $10 \times$, d $40 \times)$. Immunohistochemistry was positive for CD10 (e) and PAX8 (f). These findings are consistent with metastatic renal cell carcinoma. 
myelitis. Biopsy may be necessary to confirm the diagnosis [4].

Unfortunately, the prognosis for patients with subungual metastasis is poor. In a review of 31 cases of subungual metastases, more than $80 \%$ of the patients died either as a direct or indirect consequence of their malignancy within less than 6 months of detection of the subungual metastasis [4]. As a result, treatment of metastatic RCC is limited and often palliative [12].

In conclusion, subungual metastases, particularly from RCC, are rare. In a patient presenting with a subungual lesion, tumor metastasis to the nail unit should be included in the clinical differential diagnosis in both oncology patients and previously cancer-free individuals, as a subungual metastasis may be the first indication of a clinically silent visceral malignancy.

\section{Statement of Ethics}

Proper consent was obtained from the included patients.

\section{Disclosure Statement}

Dr. Boni E. Elewski: clinical research support (research funding provided to the University of Alabama Birmingham): Abbvie, Boehringer Ingelheim, Celgene, Incyte, Leo, Lilly, Merck, Novartis, Pfizer, Regeneron, Sun, Valeant (Ortho dermatology); consultant (received honorarium): Boehringer Ingelheim, Celgene, Leo, Lilly, Novartis, Pfizer, Sun, Valeant (Ortho dermatology). None of the above disclosed conflicts are relevant to this paper. The remaining authors have no conflicts of interest to disclose. No external or institutional funding was received for this study.

\section{References}

1 Alcaraz I, Cerroni L, Rütten A, Kutzner H, Requena L. Cutaneous metastases from internal malignancies: a clinicopathologic and immunohistochemical review. Am J Dermatopathol. 2012 Jun;34(4):347-93.

2 Brady LW, O’Neill EA, Farber SH. Unusual sites of metastases. Semin Oncol. 1977 Mar; 4(1):59-64.

3 Mueller TJ, Wu H, Greenberg RE, Hudes G, Topham N, Lessin SR, et al. Cutaneous metastases from genitourinary malignancies. Urology. 2004 Jun;63(6):1021-6.

4 Cohen PR. Metastatic tumors to the nail unit: subungual metastases. Dermatol Surg. 2001 Mar;27(3):280-93.

5 Ferringer T. Immunohistochemistry in dermatopathology. Arch Pathol Lab Med. 2015 Jan;139(1):83-105.
6 Sangoi AR, Karamchandani J, Kim J, Pai RK, McKenney JK. The use of immunohistochemistry in the diagnosis of metastatic clear cell renal cell carcinoma: a review of PAX-8, PAX-2, hKIM-1, RCCma, and CD10. Adv Anat Pathol. 2010 Nov;17(6):377-93.

7 Nibhoria S, Tiwana KK, Kaur M, Kumar S. A clinicopathological and immunohistochemical correlation in cutaneous metastases from internal malignancies: a five-year study. J Skin Cancer. 2014;2014:793937.

8 Wong CY, Helm MA, Helm TN, Zeitouni N. Patterns of skin metastases: a review of 25 years' experience at a single cancer center. Int J Dermatol. 2014 Jan;53(1):56-60.

9 Koga S, Tsuda S, Nishikido M, Matsuya F, Saito Y, Kanetake H. Renal cell carcinoma metastatic to the skin. Anticancer Res. 2000 May-Jun;20 3B:1939-40.
10 Vine JE, Cohen PR. Renal cell carcinoma metastatic to the thumb: a case report and review of subungual metastases from all primary sites. Clin Exp Dermatol. 1996 Sep;21(5):37780.

11 Lookingbill DP, Spangler N, Sexton FM. Skin involvement as the presenting sign of internal carcinoma. A retrospective study of $7316 \mathrm{can}$ cer patients. J Am Acad Dermatol. 1990 Jan; 22(1):19-26.

12 Fernández-Rueda P, Ruiz-López P, RamírezNegrín MA, Fuentes-Suárez A, ToussaintCaire S, Vega-Memije ME. [Cutaneous metastasis of renal cell carcinoma: A case report and review of the literature]. Gac Med Mex. 2015 Jul-Aug; 151(4):533-7. 